\title{
Round table on open quantum systems
}

\author{
Miguel Ángel Escobedo ${ }^{1, *}$ \\ ${ }^{1}$ Instituto Galego de Física de Altas Enerxías (IGFAE), Universidade de Santiago de Compostela. E- \\ 15782, Galicia, Spain
}

\begin{abstract}
This is a discussion about open quantum systems and its application to the study of hard probes. It reviews some of the things that were said in the corresponding round table. Views are only my own.
\end{abstract}

\section{Introduction}

In the last years, many groups have applied the formalism of open quantum systems to study quarkonium suppression [1-14] and jet quenching [15]. In my opinion, one of the reasons is that when you study these problems from the point of view of quantum field theory it is common to find the same type of equations that appear when you study other problems, for example quantum optics, using the open quantum system formalism. In other words, when we study quarkonium's evolution in a medium assuming that we know how the medium behaves what we are effectively doing is making a trace over the environment degrees of freedom.

In the formalism of open quantum system, the universe is divided in two pieces. The system of interest and the environment. The state of the system is encoded by the reduced density matrix, which is obtained making a trace over the environment degrees of freedom to the universe's density matrix. The time evolution of the reduced density matrix is ruled by the so-called master equation. When applying this formalism to the study of hard probes in heavy-ion collisions, the challenge is twofold. On one hand, we try to derive the master equation from QCD. On the other hand, it is often difficult to solve the master equation to obtain phenomenological results. I will focus on the application of the open quantum system framework to the study of quarkonium suppression, however, there also other interesting applications in other fields, as for example, jet quenching, cold atoms and cosmology.

\section{Challenges}

\subsection{More general derivations of the master equation}

Most derivations of the master equation have used either Hard Thermal Loop perturbation theory [1, 12] or pNRQCD in the limit $\frac{1}{r} \gg T$ [9]. In order to obtain more reliable predictions, we should investigate a wider range of temperature regimes and, at the same time, incorporate more non-perturbative input. Note that we observe many different quarkonium species in heavy-ion collisions (as for example, $J / \Psi, \Upsilon(1 S), \Upsilon(2 S)$...). Each species has a different radius and, therefore, they probe the medium at different scales. On top of this, the

\footnotetext{
*e-mail: miguelangel.escobedo@usc.es
} 
medium temperature changes with time as the quark-gluon plasma expands and cools down and, furthermore, depends on the impact parameter of the collision. Therefore, many different types of relations between the radius of quarkonium $r$ and the temperature $T$ are realized nowadays in heavy-ion collisions.

Regarding the combination of Non-relativistic Effective Field Theories (NREFTs) with the open quantum system framework [8-10], the results available at the moment include the derivation of the master equation in the $\frac{1}{r} \gg T$ regime and the application to phenomenology of the more restricted case $\frac{1}{r} \gg T \gg E$. From the point of view of NREFT, the case $\frac{1}{r} \gg T$ is the simpler to analyse. To generalize the nowadays available results to the case $\frac{1}{r} \sim T$ requires some new theoretical developments. The reason is the following. In a non-relativistic system, as heavy quarkonium, different well separated energy scales are present. The mass of the heavy quarks $m$ is much larger than the inverse of the typical radius, $\frac{1}{r}$, and this is much larger than the binding energy $E$. The use of NREFTs is useful for the study of this kind of systems. Starting from QCD, Non-relativistic QCD (NRQCD) [16, 17] is obtained after integrating out the scale $m$. If we also integrate out the scale $\frac{1}{r}$ we obtain potential NRQCD (pNRQCD) [18]. In the case $\frac{1}{r} \gg T$, we can perform the matching between NRQCD and pNRQCD ignoring the medium. Then, we can use pNRQCD to compute medium effects and derive the master equation. If, instead, we are considering the case $\frac{1}{r} \sim T$ then we can not ignore the medium when matching NRQCD to pNRQCD. This requires to substantially change the matching procedure.

The computation of thermal effects in real-time requires the use of the Schwinger-Keldysh contour in which one can speak of a doubling of degrees of freedom. We use a path-integral that goes from minus infinity to infinity and then goes back to minus infinity. We can call the fields on the first part of the path integral fields of type 1 (time ordered) and the rest of fields are called fields of type 2 (anti time ordered). In general, when we integrate out thermal effects, we expect to generate terms that mix both types of fields. This is a manifestation of the fact that medium effects can not always be encoded in an effective action. When dissipation is present, we need to use the more general concept of influence functional instead [19]. This is a translation to the path integral language of the observation that not all master equations are of the Liouville-von Neumann type. How does this affects the matching between NRQCD and PNRQCD? That is an open question ${ }^{1}$. In the literature, one can find several studies on how to construct an EFT including dissipation and what are the symmetries of the influence functional (see for example [22, 23]). Many of those studies are focussed on deriving an EFT for hydrodynamics and, perhaps, some of these ideas would be also useful to study quarkonium.

\subsection{Incorporating non-perturbative input}

There are two types of non-perturbative input that have been used to constraint the master equation of quarkonium. The static potential [24,25] and transport coefficients, like $\kappa$ and $\gamma$.

Regarding the static potential, it is interesting to discuss if it is possible, and how, to establish that the static potential is useful to the study of heavy quarkonium. At $T=0$, the static potential coincides with a Wilson coefficient of pNRQCD. The matching between NRQCD and PNRQCD can be performed non-perturbatively in the static limit and, since each term has a well-defined power counting in terms of $\frac{1}{m}$, the Lagrangian so-obtained can be used to describe heavy quarkonium [18]. At finite temperature, an analogous procedure can be followed as long as we only care about time-ordered correlators. The static potential

\footnotetext{
${ }^{1}$ Note that one thing that we do know is that the doubling of degrees of freedom can be ignored if we are only interested in the time-ordered correlator [20,21]. Unfortunately, then it is not possible to derive the master equation.
} 
coincides with a Wilson coefficient in pNRQCD that enters the leading-order evolution of quarkonium's time-ordered propagator. Regarding the master equation, less is known. We do not know exactly how the static potential enters the master equation of quarkonium without using perturbative arguments. To understand this we need to construct a version of pNRQCD valid for the derivation of the master equation in the $\frac{1}{r} \sim T$ regime, an issue we discussed previously.

In the limit $\frac{1}{r} \gg T \gg E$ the situation is more clear. The influence of the medium into the master equation can be encoded in two non-perturbative parameters, $\kappa$ and $\gamma[8,9] . \kappa$ coincides with the heavy quark diffusion parameter [26], useful in the study of open heavy flavour. It is related to the imaginary part of the potential and controls the strength of the dissipative (non-unitary) part of the master equation. On the other hand, $\gamma$ is related with the real part of the potential and encodes the influence of the medium into the unitary part of the master equation. Physically, it can be related to screening with the caveat that the small $r$ limit is used. We have a lot of information on $\kappa$ from lattice QCD (see [27] for a recent computation), enough to have an educated guess of the dependence of $\kappa$ with the temperature. Regarding $\gamma$, less information is available. There are determinations of $\gamma$ using the thermal mass shift of quarkonium [10], which is proportional to $\gamma\left\langle r^{2}\right\rangle$ assuming the hierarchy $\frac{1}{r} \gg$ $T \gg E$. However, it is not enough at the moment to understand how $\gamma$ depends on the temperature. An interesting question is whether $\kappa$ and $\gamma$ can be used to constraint the master equation beyond the regime $\frac{1}{r} \gg T \gg E$.

There is work that needs to be done in order to find new operators computable on lattice QCD that can bring new information about the master equation when $\frac{1}{r} \sim T$. It is possible that these operators have a complicated time-ordering because of the mixing between the two branches of the Schwinger-Keldysh contour. In fact, this kind of operators emerge on the study of the evolution of a static pair in a plasma [28]. It is not clear how these operators can be computed on lattice QCD, but that does not mean that it can not be done. The issue is that only computations in Euclidean space can directly be performed on lattice QCD, then, using analytic continuation Minkowski space quantities can be obtained. It needs to be understood how to do the analytic continuation in order to compute operators mixing the two branches of the contour.

\subsection{Recombination}

One of the phenomena that affect the number of quarkonium bound states in a heavy-ion collision is recombination $[29,30]$, the process in which two heavy quarks meet inside of the medium and form a new bound state. We can distinguish two kinds of recombination, the correlated and the uncorrelated one. In correlated recombination the two heavy quarks were part of a bound state previously, this bound state dissociated but after some time the heavy quarks recombine again. This is the kind of recombination that appears when we study the dilute limit. Uncorrelated recombination means that the heavy quarks that recombine did not belong to the same bound state before or that they might never have been in a bound state. This kind of recombination increases with the number of heavy quarks in the collision and is believed to be a leading-order effect for charmonium at LHC.

Correlated recombination is one of the main ingredients of the open quantum system framework. In fact, correlated recombination compensates the effect of the imaginary part of the potential and make the master equation trace preserving. However, it is challenging to incorporate uncorrelated recombination. Until now, two different strategies have been followed in the literature. The use of semi-classical approximations and the molecular chaos hypothesis. 
Using the Langevin equation in the Abelian limit [11] or Langevin-like equations in QCD [12], systems with many heavy particles can be simulated. This is due to the fact that classical equations are much less costly to simulate that quantum master equations. One of the problems identified in QCD is that the applicability region seems to be much narrower than the corresponding QED case, however, there have been recent studies trying to improve this situation [31]. Both in QCD and QED, uncorrelated recombination has been observed, showing that semi-classical equations allow to qualitatively understand this phenomenon. However, it is challenging to understand to which quarkonium state the newly formed bound state correspond. This is because in order to define what is, for example, a 1S state and what is not we need to talk about the wave function, an object that disappears when making the semiclassical approximation.

Another approach is to use the molecular chaos hypothesis [4]. In the context of Boltzmann equations, this hypothesis means that the 2-particle distribution function is assumed to be equal to the product of two 1-particle distribution functions. For the case of quarkonium, this means that the distribution of octets is assumed to be proportional to a product of the uncorrelated distribution of individual heavy quarks. Using this hypothesis, the Boltzmann equation can be solved and a good agreement with experimental data is found. An open question is then to find a scheme in which this hypothesis becomes the leading order result in a controlled approximation. This is still an open question.

At the moment, most of the theoretical computations use the dilute limit. In an effective way, this is equivalent to assuming a system formed by a heavy quark, a heavy antiquark and a medium without other heavy particles. Perhaps, a study considering two pairs of heavy particles in detail would give some useful insight on the phenomenon of recombination from the point of view of open quantum systems.

\section{References}

[1] Y. Akamatsu, Phys. Rev. D 91, 056002 (2015), 1403.5783

[2] A. Rothkopf, Phys. Rept. 858, 1 (2020), 1912.02253

[3] Y. Akamatsu (2020), 2009. 10559

[4] X. Yao, T. Mehen, Phys. Rev. D 99, 096028 (2019), 1811.07027

[5] X. Yao, B. Müller, Phys. Rev. D 100, 014008 (2019), 1811.09644

[6] X. Yao, W. Ke, Y. Xu, S.A. Bass, B. Müller, JHEP 01, 046 (2021), 2004.06746

[7] X. Yao, Int. J. Mod. Phys. A 36, 2130010 (2021), 2102 . 01736

[8] N. Brambilla, M.A. Escobedo, J. Soto, A. Vairo, Phys. Rev. D 96, 034021 (2017), 1612.07248

[9] N. Brambilla, M.A. Escobedo, J. Soto, A. Vairo, Phys. Rev. D 97, 074009 (2018), 1711.04515

[10] N. Brambilla, M.A. Escobedo, A. Vairo, P. Vander Griend, Phys. Rev. D 100, 054025 (2019), 1903.08063

[11] J.P. Blaizot, D. De Boni, P. Faccioli, G. Garberoglio, Nucl. Phys. A 946, 49 (2016), 1503.03857

[12] J.P. Blaizot, M.A. Escobedo, JHEP 06, 034 (2018), 1711.10812

[13] J.P. Blaizot, M.A. Escobedo, Phys. Rev. D 98, 074007 (2018), 1803.07996

[14] R. Katz, P.B. Gossiaux, Annals Phys. 368, 267 (2016), 1504.08087

[15] V. Vaidya, X. Yao, JHEP 10, 024 (2020), 2004.11403

[16] W.E. Caswell, G.P. Lepage, Phys. Lett. B 167, 437 (1986)

[17] G.T. Bodwin, E. Braaten, G.P. Lepage, Phys. Rev. D 51, 1125 (1995), [Erratum: Phys.Rev.D 55, 5853 (1997)], hep-ph/9407339 
[18] N. Brambilla, A. Pineda, J. Soto, A. Vairo, Nucl. Phys. B 566, 275 (2000), hep-ph/9907240

[19] R.P. Feynman, F.L. Vernon, Jr., Annals Phys. 24, 118 (1963)

[20] N. Brambilla, J. Ghiglieri, A. Vairo, P. Petreczky, Phys. Rev. D 78, 014017 (2008), 0804.0993

[21] M.A. Escobedo, J. Soto, Phys. Rev. A 78, 032520 (2008), 0804.0691

[22] A. Baidya, C. Jana, R. Loganayagam, A. Rudra, JHEP 11, 204 (2017), 1704.08335

[23] M. Crossley, P. Glorioso, H. Liu, JHEP 09, 095 (2017), 1511.03646

[24] Y. Burnier, O. Kaczmarek, A. Rothkopf, Phys. Rev. Lett. 114, 082001 (2015), 1410.2546

[25] D. Bala, O. Kaczmarek, R. Larsen, S. Mukherjee, G. Parkar, P. Petreczky, A. Rothkopf, J.H. Weber (2021), 2110. 11659

[26] J. Casalderrey-Solana, D. Teaney, Phys. Rev. D 74, 085012 (2006), hep-ph/0605199

[27] N. Brambilla, V. Leino, P. Petreczky, A. Vairo, Phys. Rev. D 102, 074503 (2020), 2007.10078

[28] M.A. Escobedo, Phys. Rev. D 103, 034010 (2021), 2010. 10424

[29] P. Braun-Munzinger, J. Stachel, Phys. Lett. B 490, 196 (2000), nucl-th/0007059

[30] R.L. Thews, M. Schroedter, J. Rafelski, Phys. Rev. C 63, 054905 (2001), hep-ph/0007323

[31] S. Delorme, also on these proceedings 